Rev. Elev. Méd. vét. Pays trop., 1979, 32 (4) : 361-369.

\title{
Trématodes hépato-biliaires de divers ruminants sauvages d'Afrique centrale
}

\author{
par M. GRABER $\left(^{*}\right)$ et J. THAL
}

\begin{abstract}
RESUMÉ
Les auteurs signalent la présence en Afrique centrale (Tchad, République Centrafricaine) de deux trématodes parasites des canaux biliaires et de la vésicule de divers ruminants sauvages.

Le premier, Dicrocoelium hospes Looss, 1907, n'a été rencontré que chez le buffle (16 p. 100).

Le second, Fasciola gigantica Cobbold, 1855, affecte surtout les Cobs -cob de Buffon et cob defassa - (35 p. 100) et les buffles (32 p. 100), plus rarement les bubales et les hippotragues. Les autres espèces (12) ne sont pas atteintes.

Les auteurs donnent quelques renseignements sur la répartition géographique de deux helminthes, ainsi que sur leur rôle pathogène.
\end{abstract}

\section{INTRODUCTION}

Dans le cadre d'une étude générale sur la pathologie de la faune sauvage d'Afrique centrale (République Centrafricaine (R.C. A.), Tchad, Nord Cameroun), le parasitisme des ruminants a retenu particulièrement l'attention. Une première liste d'helminthes a pu être dressée au Tchad où les enquêtes ont commencé dès $1954(10,7)$.

Ultérieurement, c'est-à-dire entre 1969 et 1972, d'autres collections ont été rassemblées dans le centre et dans le sud du Tchad (Drs PROVOST, CHAILLOUX et BORREDON), dans le nord du Cameroun (Dr MACON) et dans toute la zone cynégétique de l'est de la République Centrafricaine (22).

De nombreux parasites ont été recueillis. Certains qui sont susceptibles d'infester à la fois les herbivores domestiques et les herbivores sauvages (10) présentent, de ce fait, un grand

(*) Service de Parasitologie, Ecole Nationale Vét. de Lyon, Marcy L'Etoile, 69260 Charbonnières les Bains.

I. E. M. V. T., 10, rue Pierre-Curie, 94700 Maisons Alfort, France. intérêt : c'est le cas notamment des Distomes (Trematoda, Fascioloidea) localisés au foie et à la vésicule biliaire.

\section{MATERIEL ET MÉTHODE}

\section{Matériel}

Cent cinquante-sept autopsies complètes ont été effectuées se répartissant ainsi :

Bubalus (Syncerus) caffer, Sparrman, le Buffle : 81 (R. C. A., 71 ; Tchad, 9 : Nord Cameroun, I).

Alcelaphus lelwel, Heuglin, le Bubale : 15 (R. C. A., 13 ; Tchad, 1 ; Nord Cameroun, 1).

Kobus defassa, Rüppel, le Waterbuck ou Cob defassa : 11 (R. C. A., 7 ; Tchad, 4).

Hippotragus equinus, Desmarest, l'Hippotrague : 11 (R. C. A., 7 ; Tchad, 3 : Nord Cameroun, 1).

Kobus cob, Erxleben, le Cob de Buffon: 10 (R. C. A., 5 ; Tchad, 5).

Ourebia ourebi, Zimmerman, l'Ourébi : 8 (R. C. A., 3 ; Tchad, 1 ; Nord Cameroun, 4). 
Redunca redunca nigeriensis, Blaine, le Redunca : 5 (R. C. A., 2 ; Tchad, 3).

Sylvicapra grimmia, Linné, le Céphalophe couronné : 5 (R. C. A., 3 ; Nord Cameroun, 2).

Tragelaphus scriptus, Pallas, le Guib: 4 (R. C. A., 3 ; Tchad, 1).

Taurotragus derbianus, Gray, l'Eland de Derby : 4 (R. C. A., 3 ; Nord Cameroun, 1).

Damaliscus korrigum, Ogilby, le Damalisque : 2 (R. C. A., 1 ; Tchad, 1).

Gazella ruffrons, Gray, la Gazelle corinne : 1 (Tchad).

\section{Méthode}

Les trématodes ont été déterminés après coloration au carmin chlorhydrique et montage au baume du Canada.

\section{LES PARASITES}

Deux espèces ont été identifiées dans les canaux biliaires, la vésicule et le canal cholédoque :

1. Fasciola gigantica Cobbold, 1855 (Fascioloidea : Fasciolidae)

Hôtes: buffle, cob defassa, cob de Buffon, bubale et hippotrague.

Il s'agit d'un trématode de grande taille (25-75 × 3-12 mm), de couleur grisâtre, allongé, à bords presque parallèles. La cuticule est couverte d'épines, surtout sur la face ventrale.

L'extrémité antérieure (fig. $\mathrm{n}^{\circ} 1$ ) porte un prolongement, le cône céphalique, séparé du reste du corps par un élargissement peu prononcé.

La ventouse buccale est petite et terminale. La ventouse ventrale est plus grande que la ventouse buccale. Elle est arrondie et fait saillie à la base du cône céphalique.

Il existe un pharynx et un court cesophage. Les ramifications caecales, plus nombreuses du côté interne (fig. $\mathrm{n}^{\circ} 2$ ), s'étendent jusqu'à l'extrémité postérieure du ver qui est obtuse.

Le pore génital médian est situé immédiatement en avant de l'acétabulum. Les testicules qui occupent la plus grande partie de la moyenne du corps sont sinueux et repliés sur eux-mêmes en forme de pelote glomérulaire. Le rapport longueur des testicules/longueur du corps varie de 3,2 à $3,9 / 5$.

L'ovaire, également ramifié, est placé en avant des testicules. Entre ceux-ci et la ventouse postérieure, s'étend l'utérus disposé en rosette. Les glandes vitellogènes constituent dans les champs latéraux deux larges bandes parallèles réunies en région postérieure. Les œufs de couleur «jaune bouton d'or» et pourvus d'un opercule polaire renferment une masse moruliforme emplissant toute la coque $(160-200 \times 90$ $105 \mu)$.

Cette description sommaire s'applique principalement aux Fasciola des ruminants domestiques (Zébus du Tchad, de Madagascar et de la République populaire du Congo; moutons du Tchad).

Chez les herbivores sauvages, le trématode, bien que possédant les mêmes caractères morphologiques, est beaucoup plus polymorphe, comme le montre la figure $n^{\circ} 3$.

Quelques dimensions ont été relevées (moyenne) :

TABLEAU $\mathrm{N}^{\circ} \mathrm{I}$

\begin{tabular}{|c|c|c|c|c|}
\hline . & $\begin{array}{l}\text { Rapport } \\
\text { Longueur testicules/ } \\
\text { 1ongueur du corps }\end{array}$ & $\begin{array}{l}\text { Longueur } \\
\text { (mm) }\end{array}$ & $\begin{array}{c}\text { Largeur } \\
(\mathrm{mm})\end{array}$ & $\begin{array}{c}0 \text { e u } f s \\
\left(\begin{array}{l}(-) \\
\text { s }\end{array}\right.\end{array}$ \\
\hline Zébu, Tchad & $3,2-3,4 / 5$ & 46 & 8,6 & $163 \times 99$ \\
\hline Buffle, R.C.A. & $3,3-3,5 / 5$ & 30,5 & 5 & $157 \times 86$ \\
\hline $\begin{array}{l}\text { Waterbuck } \\
\text { - Tchad } \\
\text { - R.C.A. }\end{array}$ & $\begin{array}{l}3-3,4 / 5 \\
3,3-3,4 / 5\end{array}$ & $\begin{array}{l}34,1 \\
30,5\end{array}$ & $\begin{array}{l}12,7 \\
13,7\end{array}$ & $\begin{array}{l}155 \times 85 \\
164 \times 91\end{array}$ \\
\hline $\begin{array}{l}\text { Cob de Buffon } \\
\text { - Tchad } \\
\text { - R.C.A. }\end{array}$ & $\begin{array}{c}2,9-3 / 5 \\
-\end{array}$ & $\begin{array}{l}29,1 \\
28,2\end{array}$ & $\begin{array}{l}9,8 \\
8\end{array}$ & $\begin{array}{l}146 \times 90 \\
158 \times 82\end{array}$ \\
\hline
\end{tabular}




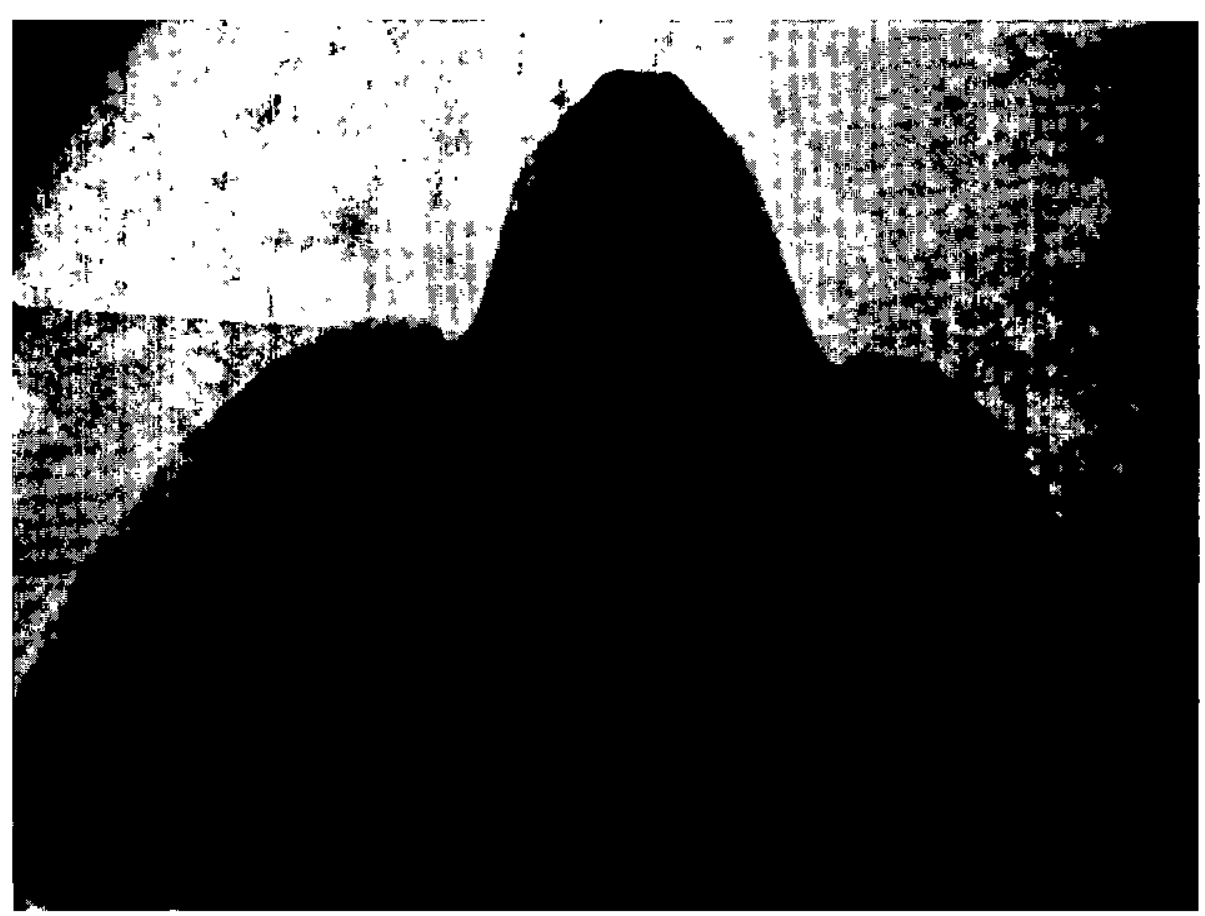

Fig. no 1. - Fasciola gigantica - Cône céphalique - Cob de Buffon, Tchad $(\times 11)$.

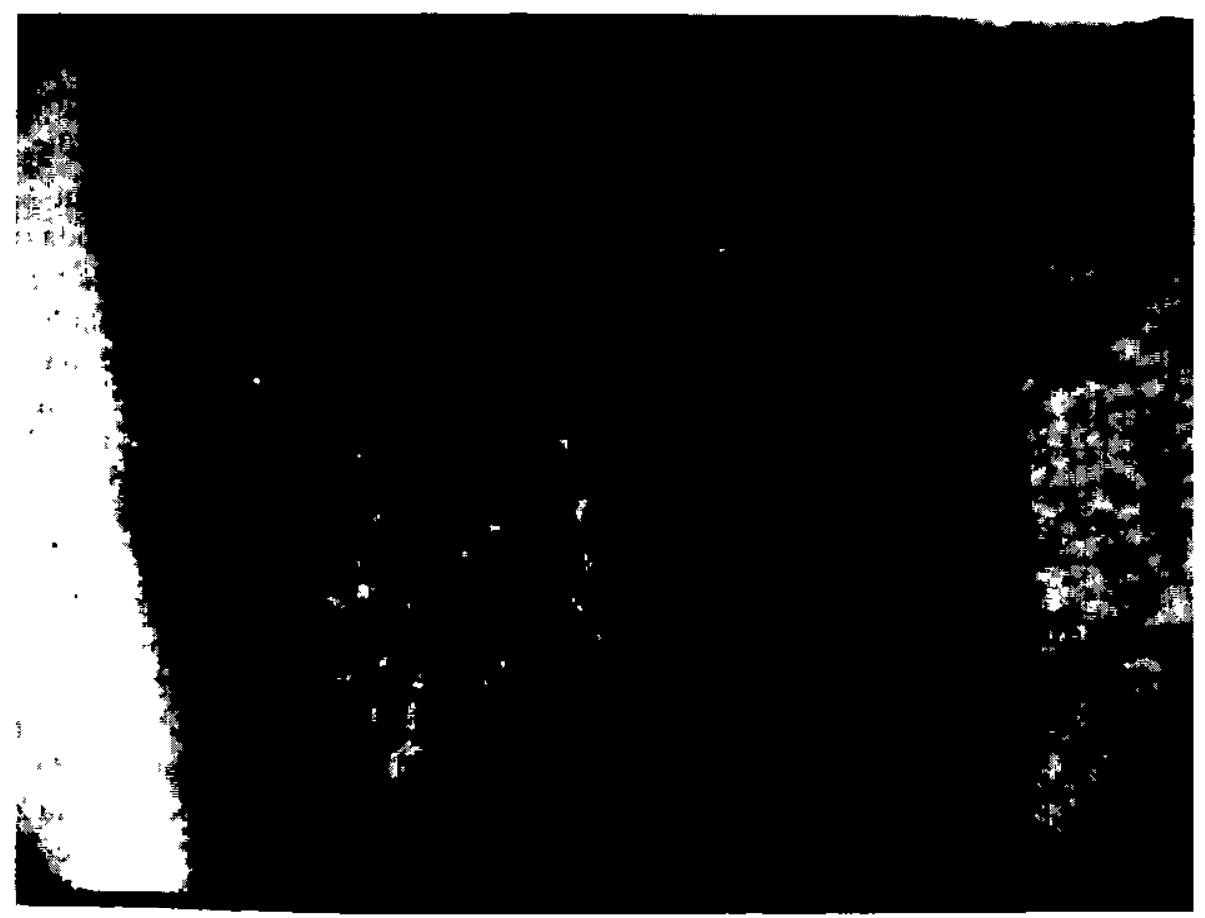

Fig. $n^{\circ}$ 2. - Fasciola gigantica - Ramifications caecales internes - Cob defassa, R. C. A. $(\times 11)$, 


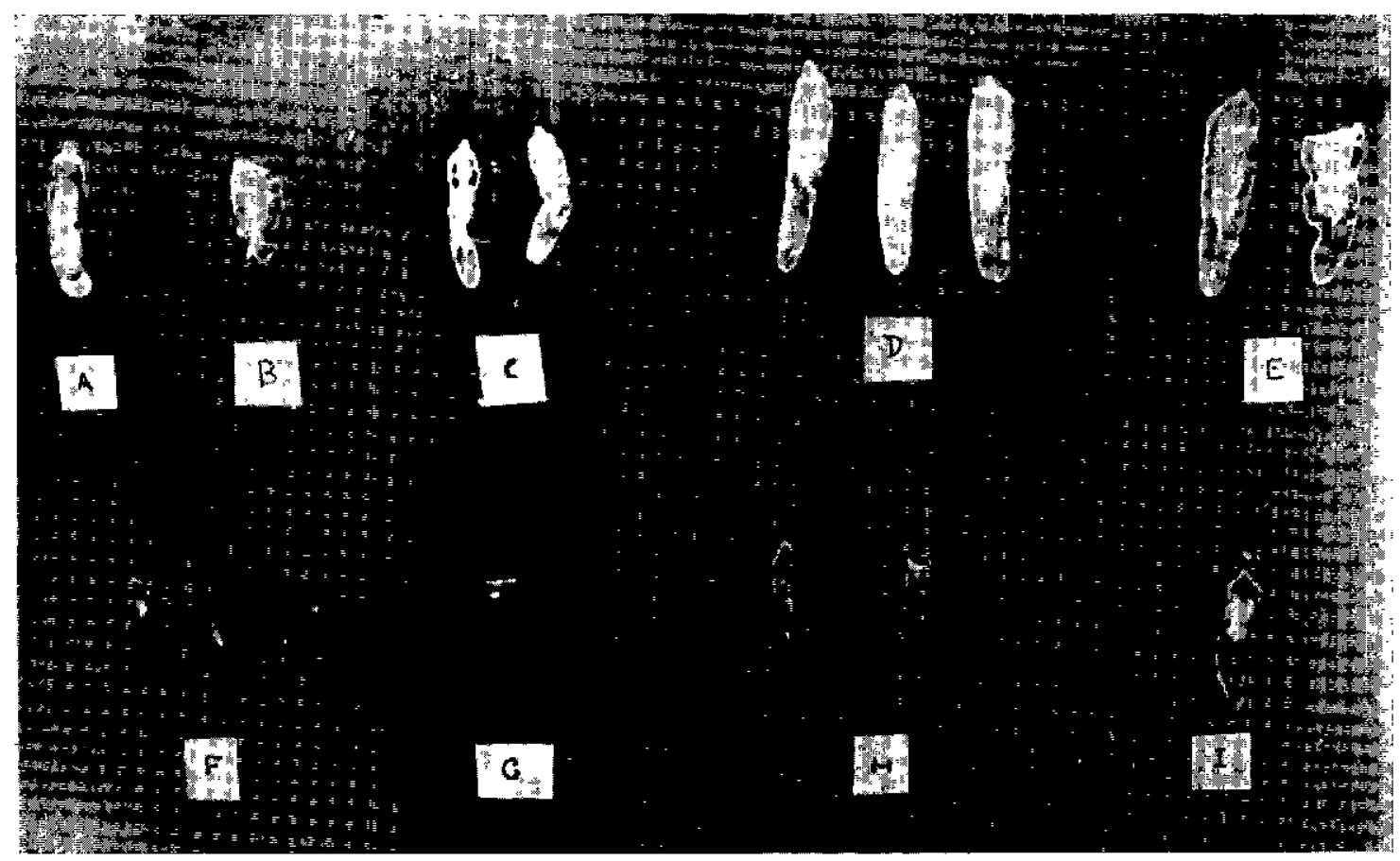

Fig. n $\mathrm{n}^{0}$ 3. - Fasciola gigantica - Aspect général
A. Bœuf Madagascar.
B. Mouton N'Djaména (Tchad).
C. Zébu - infestation expérimentale (168 jours) - N'Djaména (Tchad).
D. Zébu - Abattoir de N'Djaména (Tchad).
E. Cob defassa, Tchad.
F. Cob defassa, R. C. A.
G. Cob de Buffon, R. C. A.
H. Buffle et Phacochère, R. C. A.
I. Fasciola nyanzae, Hippopotame, Tchad.

\section{Dicrocoelium hospes Looss, 1907 (Fas- cioloidea : Dicrocoeliidae)}

Hôte : Buffle.

Ce trématode a été redécrit récemment $(11,20)$. Les Dicrocoelium des buffles d'Afrique centrale sont un peu plus courts et leurs organes moins développés (sauf le pharynx et les testicules) que ceux des moutons ou des zébus originaires de la même région. Ce fait avait déjà été constaté en République populaire du Congo (11).

\section{COMMENTAIRES}

1. En Afrique centrale (Tchad, R. C. A. $\left({ }^{*}\right)$ ), Fasciola gigantica avait déjà été observée chez le buffle, le cob de Buffon, le cob defassa et le bubale (7).

(*) Aucun des animaux autopsiés dans le Nord du Cameroun n'était porteur de Fasciola gigantica.
La présente enquête permet d'ajouter à cette liste l'hippotrague qui est un hôte nouveau.

Fasciola gigantica, en Afrique tropicale, affecte un grand nombre d'artiodactyles sauvages appartenant à la famille des bovidés (17). Outre les espèces précédemment indiquées, on la rencontre également chez le céphalophe couronné, l'impala, la gazelle de Thomson, le puku, le redunca, le damalisque, l'éland de Derby et le koudou, soit une cinquantaine d'observations sur l'ensemble du continent africain (12).

Les enquêtes ont été effectuées dans le sud du Soudan (15), en Uganda dans diverses réserves $(5,12)$, en Tanzanie $(12,18)$ dans le Parc de Serengeti ainsi que dans le Nord du pays (14), au Kenya $(12,23)$, en Zambie (16), au Zimbabwé $(2,12,16)$, au Rwanda (6) et au Zairre (21).

Outre Fasciola gigantica, il existe en Afrique noire une seconde espèce, Fasciola hepatica Linné, 1758 qui colonise les hautes terres de l'Est africain. En Ethiopie, ce distome a effec- 
tivement été recueilli dans les canaux bilıaires du céphalophe de Grimm (9). Ailleurs - et notamment au Zaïre -, sa présence est douteuse et les identifications faites il y a déjà 50 ans demandent à être de nouveau soigneusement vérifiées $(19,21)$.

\section{Plusieurs foyers ont pu être délimités (carte)}

\subsection{Bassin du Chari}

- En R. C. A., celui du Bahr Aouk (209 B a) $\left(^{*}\right)$ un cob de Buffon et deux buffles : celui des Rivières Vakaga et Kapa (21 9 B b ;21 8 D d; $218 \mathrm{D}$ a), un bubale, un hippotrague, un cob defassa et deux buffles et celui du Bamingui $(207 \mathrm{C} \mathrm{b})$, un cob de Buffon.

- Au Tchad, celui de l'Aouk (19 9 A a), un cob de Buffon et celui de la moyenne vallée du Chari (17 $10 \mathrm{~A} \mathrm{c})$, un buffle.

\subsection{Bassin de l'Oubanghui, affluent du Zaïre}

- Celui de la haute Kotto (23 7 A b ; 237 $\mathrm{A} \mathrm{d} ; 237 \mathrm{C} \mathrm{b} ; 237 \mathrm{D} \mathrm{c} ; 238 \mathrm{~B} \mathrm{~b}$ ), un Cob defassa et 10 buffles.

- Celui des Rivières Dji et Mbali (236 A d ; 236 B c ; $236 \mathrm{C} \mathrm{d}$ ), 9 buffles.

- Celui de la rivière Chinko (24 $5 \mathrm{C} \mathrm{b}$; 245 A d ; 246 B d), 4 buffles.
L'aire de répartition de Fasciola gigantica couvre donc les deux bassins du Chari et de l'Oubanghui, du centre du Tchad jusqu'à la frontière du Zaïre, c'est-à-dire l'ensemble de la zone cynégétique d'Afrique centrale.

Plusieurs de ces foyers (Bahr Aouk, Rivières Kapa et Wakaga, Bamingui) se trouvent dans des régions où le contact entre ruminants domestiques atteints de distomatose et ruminants sauvages est possible. En effet, ces zones qui peuvent être, à certaines époques, envahies par des animaux venus clandestinement du Tchad (22) sont, en outre, traversées par deux routes d'importation du bétall (carte) : celle qui va de Birao à Bengassou par Ouadda et Bria et, plus à l'Ouest, celle qui va de Sahr (Tchad) à Bangui par N'Délé, Fort Crampel et Fort Sibut.

Les autres foyers (Dji, Mbali, Haute Kotto, Chinko) sont plus éloignés des pistes fréquentées par les animaux domestiques et l'on peut penser que la dissémination des distomes est le fait de certains ruminants sauvages, surtout le buffle. La même observation a été faite en Uganda (4).

3. Les taux d'infestation varient sensiblement d'une espèce à l'autre. Sur un total de trois cent douze ruminants autopsiés, entre 1954 et 1972, au Tchad, au Nord Cameroun et en République centrafricaine, la répartition est la suivante :

TABLEAU $\mathrm{N}^{\circ} \mathrm{II}$

\begin{tabular}{|c|c|c|c|}
\hline \multirow{2}{*}{$\begin{array}{l}\text { Nombre d'animaux } \\
\text { autopsiés et espèces }\end{array}$} & \multicolumn{3}{|c|}{ Nombre d'animaux parasités et pourcentage d'infestation } \\
\hline & Total & Tchad & R.C.A. \\
\hline Buffles : 88 & $29(32$ p. 100$)$ & 1 sur 14 & $\begin{array}{c}28 \text { sur } 74 \\
(37,8 \text { p. } 100)\end{array}$ \\
\hline Bubales : 34 & $2(6,1 \mathrm{p} .100)$ & 1 sur 21 & 1 sur 13 \\
\hline Cobs de Buffon : 20 & $4(\mathrm{~s}$ p. 100$)$ & 3 sur 15 & 1 sur 5 \\
\hline Cobs defassa : 25 & $12(40$ p. 100$)$ & $B$ sur 18 & 4 sur 7 \\
\hline Hippotrague : 20 & $1(5 \mathrm{p} .100)$ & 0 sur 13 & 1 sur 7 \\
\hline
\end{tabular}

L'autopsie des espèces suivantes s'est révélée totalement négative : éland de Derby $\left(4\left(^{* *}\right)\right.$ ), damalisque (9), céphalophe couronné (7), ouré-

(*) Coordonnćes préconisćes par l'inter-african Bureau of Animal Health (I. B. A. H.) qui utilise des carrés délimités par les longitudes et par les latitudes.

(**) Nombre d'autopsies. bi (13), redunca (10), gazelle dorcas (36), gazelle corinne (21), gazelle dama (9), oryx (9), addax (1), grand koudou (2), guib (5).

En Afrique centrale, les cobs (cob de Buffon et waterbuck) sont donc les herbivores les plus atteints, immédiatement suivis des buffles et, loin derrière, des bubales et des hippotragues. 


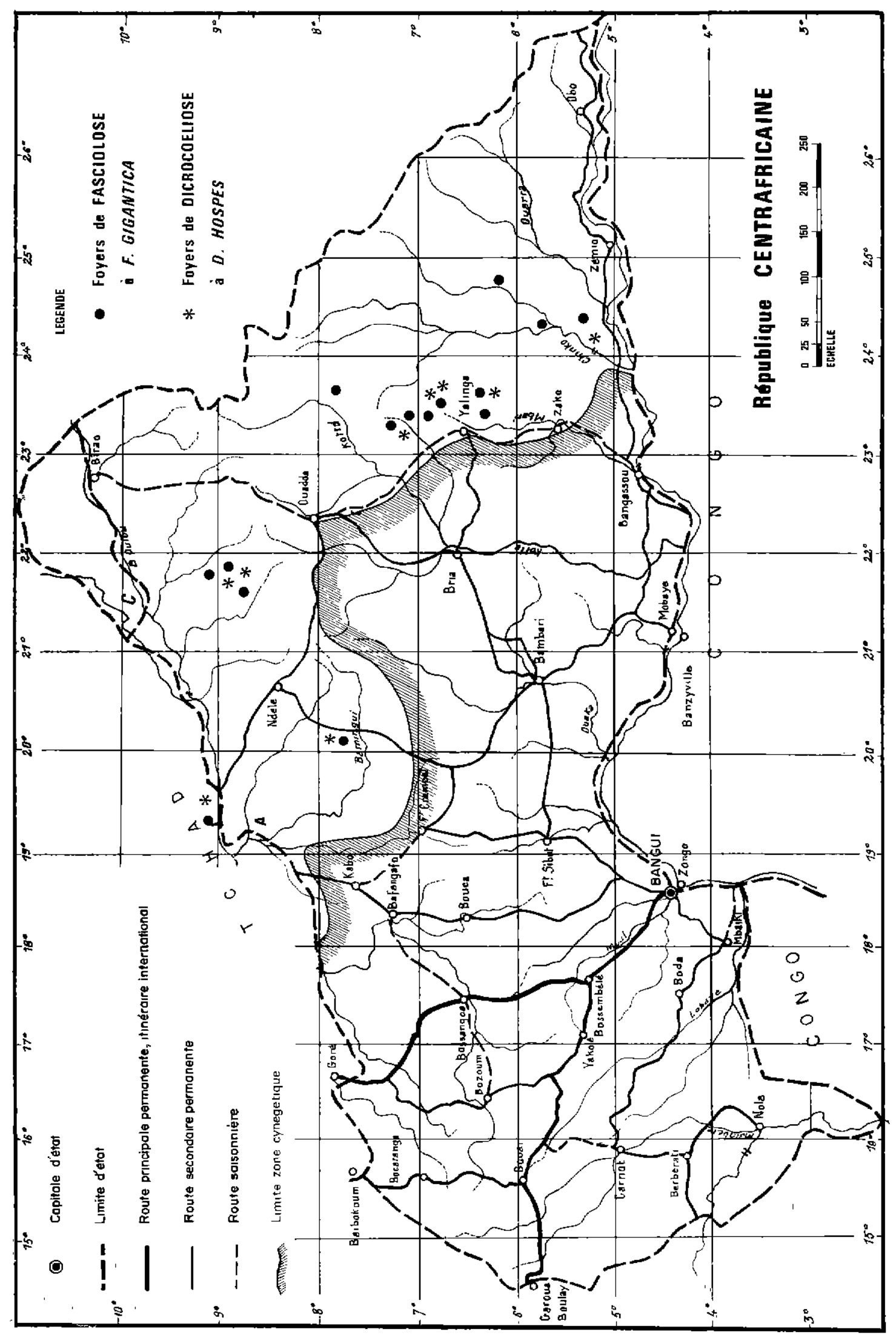


Dans d'autres régions d'Afrique, notamment en Uganda, la distomatose affecte surtout les buffles $(1,12)$ avec des taux de $58-60$ p. 100, les cobs (46-47 p. 100$)$ et les bubales $(42,5-$ 47 p. 100). Ailleurs, l'infestation des buffles est négligeable, particulièrement au parc de Serengeti en Tanzanie et au Parc Elisabeth en Uganda (12).

En ce qui concerne les autres espèces de ruminants, il est remarquable de constater que les résultats obtenus en Afrique centrale correspondent à peu de choses près à ceux d'Afrique orientale $(1,3,12)$ : dans ces régions, la distomatose hépato-biliaire est rarissime chez les antilopinés, les cephalophinés et les hippotraginés (Gazelles diverses, ourébis, céphalophes et impalas), un peu plus fréquente - moins de 10 p. 100 - chez certains alcelaphinés (damalisque, gnou) et tragelaphinés (éland, grand koudou).

On a cherché à expliquer ces différences:

- soit par la possibilité qu'auraient certains herbivores de se montrer réfractaires à toute infestation par Fasciola gigantica;

- soit par l'influence du milieu, les habitudes alimentaires et le mode d'abreuvement des animaux.

Les raisons invoquées ne sont pas toutes convaincantes et il est nécessaire de poursuivre les recherches au laboratoire et sur le terrain avant de tirer des conclusions définitives.

4. Le niveau de l'infestation varie d'une espèce à l'autre. Ainsi, la moyenne du nombre de Fascioles recueillies dans les canaux biliaires, le canal cholédoque et la vésicule qui est une localisation essentielle à ne pas négliger (I) est de 10 chez les cobs de Buffon et de 12,4 chez les waterbuck.

Chez les buffles, il n'en est pas de même et la moyenne du nombre de douves est de 30 par animal. Dans 75 p. 100 des cas, les lésions de cholangite et de cirrhose sont discrètes et l'état général des animaux ne semble pas affecté outre mesure. Sur le quart restant, les lésions sont importantes, voire massives (de cent à quatre cents douves) et les buffles autopsiés sont, la plupart du temps, maigres et fortement anémiés.

Les jeunes de moins de 5 ans sont plus atteints que les adultes: 60 p. 100 contre 45 p. 100.

Les conséquences de cet état de choses sont difficilement chiffrables. Des cas mortels de distomatose n'ont été signalés qu'en Uganda, dans le Parc du lac Mcllwaine chez l'éland, le grand koudou et le gnou (2).

Les ruminants sauvages - comme les ruminants domestiques - hébergent très souvent un grand nombre de parasites internes et externes associés (13). Dans ces conditions, il est pratiquement impossible d'établir le rôle exact des distomes hépato-biliaires. De toutes les façons, le polyparasitisme chronique se manifeste plus par des pertes de poids et par des retards de croissance que par des signes cliniques visibles.

5. En matière d'épidémiologie, sur une période de 18 mois (janvier 1970 à juin 1971), la présence en juin et en juillet de nombreuses douves immatures dans le foie laisse supposer que - comme au Tchad (11) - l'infestation des buffles de l'Est de la R. C. A. se produit au milieu et à la fin de la saison sèche.

6. Dicrocoelium hospes n'affecte que le buffle aussi bien en R. C. A., qu'au Tchad (11). Le pourcentage total d'animaux infestés est de 16 p. 100 (6 sur 14 au Tchad et 8 sur 74 en R. C. A.). Dans 50 p. 100 des cas, (en R. C. A., principalement), Dicrocoelium hospes est associé à Fasciola gigantica.

Le nombre de douves dans les canaux et dans la vésicule est, en général, faible (moins de 50). Un seul buffle, très maigre, hébergeait plusieurs milliers de Dicrocoelium. Comme chez le zébu (11), les adultes de plus de 5 ans sont plus atteints que les jeunes. En Afrique centrale, le parasite a la même répartition géographique que Fasciola gigantica.

\section{CONCLUSIONS}

Une série d'enquêtes effectuées entre 1954 et 1972 sur plus de trois cents ruminants sauvages du Tchad, du Nord Cameroun et de la République centrafricaine a permis de mettre en évidence, dans les canaux biliaires et dans la vésicule, deux trématodes : Fasciola gigantica Cobbold, 1855 et Dicrocoelium hospes Looss, 1907.

Ce dernier n'a été rencontré que chez le buffle (16 p. 100).

Fasciola gigantica affecte, dans toute la zone cygénét1que d'Afrique centrale, les cobs (cob de Buffon et cob defassa), le buffle, le bubale et l'hippotrague qui est un hôte nouveau.

Les herbivores les plus atteints sont les cobs 
(35 p. 100), immédiatement suivis des buffles (32 p. 100) et, loin derrière, des bubales et des hippotragues. Les autres herbivores examinés (douze espèces au total) n'hébergeaient aucun distome.

Dans l'est de la République centrafricaine, Fasciola gigantica semble se maintenir en dehors de toute intervention de ruminants domestiques parasités.

La fasciolose hépato-biliaire paraît, dans l'ensemble, assez bien supportée, sauf chez le buffle où, dans 25 p. 100 des cas, on observe des lésions hépatiques massives accompagnées d'une baisse sensible de l'état général.

\section{SUMMARY}

\section{Liver flukes from wild ruminants in Central Africa}

In Central Africa (Chad, Centrafrican Republic, North Cameroon), a survey was carried out from 1954 to 1972 to ascertain the incidence of liver fiukes in wild ruminants. More than three hundred animals were autopsied.

Dicrocoelium hospes was recovered only in african buffalo and Fasciola gigantica in cob and waterbuck ( 35 p. 100), african buffalo (32 p. 100), hartebeest $(6$ p. 100) and roan antelope $(5$ p. 100$)$ which is a new host. Other species (12) were not affected.

In east of Centrafrican Republic, Fasciola gigantica infection seems to be maintained in wildlife in the absence of domestic ruminants.

Generally, infected game animals show no apparent clinical effect, but, in 25 p. 100 of african buffalo, fascioliasis lesions are very important and emaciation is observed.

\section{RESUMEN}

\section{Tremátodos hepato-biliares de varios rumiantes salvajes de Africa central}

Los autores señalan la presencia en Africa central (Chad, República Centroafricana) de dos tremátodos parásitos de los canales biliares y de la vesícula de varios rumiantes salvajes.

El primero, Dicrocoelium hospes Looss, 1907 no se encontró más que en el búfalo (16 p. 100).

El segundo, Fasciola gigantica Cobbold, 1855 ataca sobretodo Adenota Kob y Kobus defassa (35 p. 100) y los búfalos (32 p. 100), más raramente Bubalus alcelaphus y Hippotragus.

No ataca otras especies.

Los autores dan algunos informes sobre la repartición geográfica de dos helmíntos asi como sobre su papel patógeno.

\section{BIBLIOGRAPHIE}

1. BINDERNAGEL (J. A.). Liver fluke Fasciola gigantica in african buffalo and antelopes in Uganda, East Africa. $J$. Wildl. Dis., 1972, 8 (4) : 315-317.

2. CHRISTIE (G. J.). Annual Report of the Director of veterinary service, Rhodesia, 1965-1966.

3. CONDY (J. B.). Observations on levels of internal parasites in free-living rhodesian wild life. I. Kudu, Tragelaphus strepsiceros (Pallas, 1866). Zool. Afr., 1972, 7 (2) : 413-418.

4. COYLE (T. J.). The epidemiology of Fasciola gigantica in cattle in Uganda protectorate. Thesis, Royal College veterinary Surgeons, London, 1961.

5. DINNIK (J. A.). On Moniezia monardi, Fuhrmann and Avitellina buechneri sp. nov. from Adenota kob thomasi (P. L. Sclater). J. Helminth., 1963, 37 (3) : 169-178.

6. FAIN (A.). Lymnaea (Radix) natalensis undussumae, Von Martens, transmetteur naturel de Fasciola gigantica, Cobbold au Congo. - Reproduction expérimentale du cycle évolutif de cette douve. Ann's Soc. belge Méd. trop., 1951, 31 (4) : 531-539.

7. GRABER (M.). Helminthes parasites de certains animaux domestiques et sauyages du Tchad. Bull. epizoot. Dis. Afr., 1969, 17 (4) : 403-428.

8. GRABER (M.). Les trématodoses hépato-biliaires et gastriques des zébus d'Afrique centrale. Rev. Elev. Méd. vét. Pays trop., 1975, 28 (3) : 311-314.
9. GRABER (M.), BLANC (Ph.), DELAVENAY (R.) Helminthes des animaux sauvages d'Ethiopie. I. - Mammifères (à paraître).

10. GRABER (M.), DOUTRE (M.), FINELLE (P.), KERAVEC (J.), DUCROZ (G.), MOKOTAINGAR (P.). Les helminthes de quelques artiodactyles sauvages appartenant aux familles des bovidés et des suidés. Ces mammifères, en République du Tchad et en R. C. A., sont-ils des réservoirs de parasites pour les animaux domestiques vivant à leur contact ? Rev. Elev. Méd. vét. Pays trop., 1964, 17 (3) : 377-419.

11. GRABER (M.), OUMATIE (O.). Existence en Afrique Equatoriale d'un important foyer de dicrocoeliose bovine et ovine à Dicrocoetium hospes Looss, 1907. Rev. Elev. Méd. vét. Pays trop., 1964, 17 (3) : 523-533.

12. HAMMOND (J. A.). Infections with Fasciola spp. in wildlife in Africa. Trop. Anim. Hlth. Prod., 1972, 4 (1) : 1-13.

13. HAMMOND (J. A.), BRANAGAN (D.). The disease factor in plans for the domestication of wild ruminants in Africa. Vet. Rec., 1973, 92 (14) : $367-$ 369.

14. MUSTAFA (M. H.). New host for Fasciola sp. in Tanzania. Vet. Rec., 1974, 94 (2) : 31.

15. MYERS (B. J.), WOLFGANG (R. W.), KUNTZ (R.). Helminth parasites from vertebrates taken in 
the Sudan (East Africa). Can. J. Zool., 1960, 38 (4) : 833-36.

16. ROTH (H. H.), DALCHOW (W.). Untersuchungen ûber den Wurmbefall von Antilopen in Rhodesien. Z. angew. Zool., 1967, 54 (2) : 203-226.

17. ROUND (M. C.). Check-list of the herminth parasites of african mammals. St Albans, Commonwealth Bureau of Helminthology, 1968, 252 p. Technical Communication No 38 .

18. SACHS (R.), SACHS (C.). A survey of parasitic infestation of wild herbivores in the Serengeti region in Northern Tanzania and the lake Rukwa region in Southern Tanzania. Bull. epizoot. Dis. Afr., $1968,16(4): 455-472$.

19. SANDGROUND (J. H.). Report on the Harward african expedition upon the african Republic of Liberia and the Belgian Congo. Pt. III. Medical and biological investigations: XXVIII. Notes and des- criptions of some parasitic helminths collected by the expedition. Contribution. Dpt. trop. Med. Inst. trop. Biol. Med. (Harward University), 1930, 5 (1) : $462-486$.

20. SEGUIN (D.). Contribution à l'étude des distomatoses hépato-biliaires des ruminants du Togo. Thèse Méd. vét. Lyon, $1975, n^{0} 64$.

21. STUNKARD (H. W.). The parasitic worms collected by the American Museum of Natural History expedition to the Belgian Congo 1909-1914. Bull. Am. Mus. nat. Hist., 1929, 58 (6) : 233-289.

22. THAL (J). Les maladies similaires à la peste bovine. Etude et lutte. Rapport Projet P. N. U. D./C. A. F. 13, Maisons-Alfort, I. E. M. V. T. 1972, 57 p.

23. URQUARTH (G. M.), HAY (D.), ZAPHIRO (D.), SPINAGE (C. A.). Some internal parasites of game animals in Kenya. E. Afr. agric. For. J., 1960, 26 (1) : 11-20. 